ISSN 1991-8631

Review

http://indexmedicus.afro.who.int

\title{
Diversité de la microflore initiale de la viande et sécurité sanitaire des consommateurs
}

\author{
C.F.A. SALIFOU ${ }^{1}$, K.C. BOKO ${ }^{1}$, G.S. AHOUNOU ${ }^{1}$, P.U. TOUGAN ${ }^{1}$, S.K. KASSA ${ }^{1}$, \\ I. HOUAGA ${ }^{1}$, S. FAROUGOU ${ }^{1}$, G.A. MENSAH ${ }^{2}$, A. CLINQUART ${ }^{3}$ et \\ A.K.I. YOUSSAO ${ }^{1 *}$ \\ ${ }^{1}$ Ecole Polytechnique d'Abomey-Calavi, Département de Production et Santé Animales, 01 BP 2009 Cotonou, \\ Bénin. \\ ${ }^{2}$ Centre de Recherches Agricoles d'Agonkanmey, Institut National des Recherches Agricoles du Bénin, \\ 01 BP 884 Recette Principale, Cotonou 01, Bénin. \\ ${ }^{3}$ Faculté de Médecine Vétérinaire de Liège, Département des Sciences de Denrées Alimentaires, \\ 20 Bd de Colonster, 4000 Liège, Belgique. \\ *Auteur correspondant, E-mail : iyoussao@yahoo.fr, issaka.youssao@epac.uac.bj; 01 BP 2009 Cotonou, \\ Bénin. Tél : 0022995285988 ou 00229979120 74, Fax: 0022921360199
}

\section{RESUME}

La microflore initiale de la viande regroupe les germes survenus de l'animal vivant jusqu'à l'obtention de la carcasse c'est-à dire jusqu'à l'habillage mais avant lavage. Cet article décrit la diversité de cette microflore, les facteurs favorisant leur multiplication et leurs conséquences sur la santé des consommateurs. Les microorganismes de surface retrouvés immédiatement après abattage sur les carcasses ont été d'abord récapitulés. Les principaux indicateurs du respect des bonnes pratiques d'hygiène dans la filière viande ont été ensuite décrits, notamment, la Flore Aérobie Mésophile, Pseudomonas, les Enterobacteriaceae et E. coli. L'implication de l'activité de l'eau, de la température, du potentiel d'oxydoréduction et du $\mathrm{pH}$ dans le développement de la microflore initiale de la viande a été présentée. L'altération des viandes, les toxiinfections alimentaires et les conséquences technologiques issues du développement de cette microflore ont été décrites. Enfin, les caractéristiques des principaux germes pathogènes de la viande ont été décrites et les normes microbiologiques de la viande appliquées dans quelques pays ont été inventoriées.

(C) 2013 International Formulae Group. All rights reserved.

Mots clés: viande, microorganisme, altération, toxi-infection, normes.

\section{INTRODUCTION}

La viande est le produit de transformation du muscle après la mort de l'animal. Sa qualité prend en compte 4 composantes : la qualité technologique, la qualité organoleptique, la qualité nutritionnelle et la qualité hygiénique. Les trois premières composantes ont été décrites chez le bovin par Salifou et al. (2013). La quatrième composante est essentiellement liée à la santé publique et constitue un critère primordial pour la sécurité sanitaire du consommateur. De ce fait, la viande ne doit contenir aucun résidu toxique, aucun parasite, ni être le siège d'un développement bactérien susceptible de produire des éléments nocifs 
(Coibion, 2008). La viande étant une denrée périssable, elle a été traditionnellement considérée comme le véhicule de nombreuses maladies d'origine alimentaire chez l'homme (Fosse et al., 2006). Sa composition en eau et en protéines de haute valeur biologique fait qu'elle est une niche très favorable au développement des microorganismes (Benaissa, 2011). De même, la transformation de l'animal vivant en carcasse puis en viande s'accompagne généralement d'une contamination de microbes au cours du procédé (Cartier, 2004). De nombreuses recherches microbiologiques ont été menées sur la viande et ont permis d'isoler par stade de production, plusieurs types de microbes selon qu'il s'agit de la viande fraîche, de la viande hachée ou des préparations à base de viande (Dennaï et al., 2001 ; Benaissa, 2011 ; Biswas et al., 2011 ; Kpodékon et al., 2013). La connaissance de ces derniers est indispensable à une bonne maîtrise de la contamination pour assurer la sécurité sanitaire des consommateurs de viandes. La présente synthèse bibliographique a pour objectif de décrire la diversité de la microflore initiale de la viande, les facteurs favorisant leur multiplication et leurs conséquences sur la santé des consommateurs.

\section{La microflore initiale de la viande}

La microflore initiale de la viande regroupe les germes survenus de l'animal vivant jusqu'à l'obtention de la carcasse c'està-dire jusqu'à l'habillage mais avant lavage (Fernandes, 2009). Ces germes proviennent soit des animaux eux-mêmes par contact direct via le cuir, les pattes, les sabots ou le tractus digestif, soit de l'eau utilisée, soit des hommes, de la méthode de travail, du milieu ou soit du matériel utilisé par contact indirect (Corry, 2007 ; Fernandes, 2009). L'abattoir est le point critique majeur de dépôt des germes sur les masses musculaires nouvellement mises à nues. Il est souhaitable de faire en sorte que les transferts des éléments pollués vers les masses musculaires jusqu'alors stériles, soient infimes. Un cuir contient environ $10^{9}$ germes $/ \mathrm{cm}^{2}$, une carcasse en fin de chaîne d'abattage est de qualité hygiénique correcte si elle contient une quantité de germes $\leq 10^{3}$ germes $/ \mathrm{cm}^{2}$ (Cartier, 2004). Les transferts des germes du cuir à la carcasse ne doivent pas dépasser plus d'une bactérie sur $10^{6}$ (Cartier, 2004).

La microflore de surface retrouvée immédiatement après abattage sur les carcasses est principalement constituée de : Micrococcus, Pseudomonas, Moraxella, Acinetobacter, Staphylococcus, Streptococcus, Bacillus, Brochothrix thermosphacta, Lactobacillus, Flavobacterium, Kurthia, les Enterobacteriaceae et les Coryneformes. On retrouve aussi une diversité de levures (genre Candida) et de moisissures (genres Penicillium, Mucor, Aspergillus, Rhyzopus) (Fernandes, 2009; Benaissa et al., 2011). Dans les régions tropicales, les germes psychrotrophes sont pratiquement absents sur les carcasses des animaux abattus (Fernandes, 2009). La majorité de ces bactéries ne contribuent pas en général à la détérioration de la viande car ils sont incapables de croître à des températures de réfrigération. Par contre, elles peuvent représenter un danger pour le consommateur en causant des toxi-infections alimentaires. Sont principalement utilisés comme indicateurs du respect des bonnes pratiques d'hygiène dans la filière viande, la Flore Aérobie Mésophile, Pseudomonas, les Enterobacteriaceae et E. coli (Ghafir et Daube, 2007).

La microflore de surface des carcasses peut être réduite si les bonnes pratiques d'hygiène et strictement les principes de la méthode HACCP sont respectés au cours de la production (Cartier, 2007). Il faut donc :

- analyser toutes les circonstances qui, de l'arrivée des animaux à l'expédition des carcasses froides, peuvent conduire à une contamination des carcasses ;

- définir des mesures préventives efficaces, aptes à limiter ces contaminations ;

- définir les systèmes de surveillance et de vérification de l'application effective des mesures préventives;

- définir des actions correctives (au niveau des postes de travail mais aussi, le cas 
échéant, sur les carcasses) si les contaminations sont jugées anormalement élevées.

De la fin de la chaîne d'abattage à la vente puis à la cuisson des viandes, l'hygiène de la manipulation en général est nécessaire pour maîtriser les développements bactériens.

\section{La flore aérobie mésophile}

La flore aérobie mésophile regroupe des microorganismes formant des colonies dénombrables après leur multiplication dans des conditions de laboratoire définies (Bonnefoy et al., 2002). Il s'agit des germes aérobies pouvant se multiplier dans des conditions ambiantes à $30{ }^{\circ} \mathrm{C}$ et ne constituant pas une famille bactérienne particulière. Cette flore regroupe des enterobacteriaceae, de Bacillus, de staphylocoques, de Pseudomonas, des bactéries lactiques ou d'autres agents éventuellement pathogènes. Leur présence audelà des limites définies peut signifier un défaut d'hygiène des procédés de fabrication. A titre d'exemple, si leur moyenne quotidienne sur les carcasses de bovins, ovins, caprins et équidés est supérieure à $5 \log$ $\mathrm{ufc} / \mathrm{cm}^{2}$, la qualité hygiénique du procédé d'abattage est insatisfaisante (UE, 2005). Audelà de $10^{7} \mathrm{ufc} / \mathrm{g}$, ces germes entraînent un état de putréfaction de la viande (Ghafir et Daube, 2007). Leur forte charge dans l'aliment peut également être due à une conservation à des températures trop élevées, sauf lorsqu'il s'agit de bactéries psychrotrophes (par exemple les bactéries lactiques, Pseudomonas, Listeria, Yersinia) (Ghafir et Daube, 2007).

\section{Pseudomonas}

Le genre Pseudomonas est constitué de bacilles Gram négatifs, droits ou légèrement incurvés, ayant une taille de 0,5 à 1,0 $\mu \mathrm{m}$ de diamètre sur 1,5 à $5,0 \mu \mathrm{m}$ de longueur, aérobies stricts, oxydase positifs, non sporulés et généralement mobiles grâce à une ciliature polaire (Figure1). Certains produisent des pigments hydrosolubles fluorescents, de couleur jaune-vert qui ont un rôle de sidérophores. La plupart des espèces sont psychrotrophes. Leur croissance est possible entre $4{ }^{\circ} \mathrm{C}$ (voire moins) et $43{ }^{\circ} \mathrm{C}$ (Euzéby, 2007). Les Pseudomonas sont ubiquistes et peuvent vivre dans des niches écologiques très diverses. Peu virulentes, plusieurs souches sont des pathogènes opportunistes pour l'homme et des agents d'altération des viandes, poissons et produits laitiers. Les espèces les plus fréquemment rencontrées chez l'homme sont Pseudomonas aeruginosa, P. fluorescens, $P$. putida et $P$. stutzeri (Euzéby, 2007). Leur présence au niveau des chaînes d'abattage et en particulier dans les chambres froides constitue une source permanente de contamination des viandes. Pseudomonas est principalement utilisé comme indicateur d'altération des viandes fraîches et du lait (Bailly et al., 2012).

\section{Les Enterobacteriaceae}

Les Enterobacteriaceae ou entérobactéries appartiennent à une famille de courts bâtonnets Gram négatifs, de 0,3 à 1,0 $\mu \mathrm{m}$ de diamètre sur 1,0 à $6,0 \mu \mathrm{m}$ de longueur, dont certains sont mobiles au moyen de flagelles péritriches et d'autres immobiles. Toutes les espèces sont anaérobies facultatifs, fermentent le glucose et sont oxydasenégatives. Il s'agit d'un groupe biochimiquement et génétiquement apparenté, présentant une grande hétérogénéité du point de vue écologie, hôtes et potentiel pathogène pour l'homme, les animaux, les insectes et les plantes (Ghafir et Daube, 2007). Cette famille inclut plusieurs genres et espèces de bactéries pathogènes intestinales (Shigella, Salmonella et les souches pathogènes de Yersinia et d'E. coli). Elle comprend également de nombreux genres présents naturellement dans l'environnement, y compris sur les plantes, sans être d'origine fécale ni associés à des maladies d'origine alimentaire (Ray, 2001). Parmi les entérobactéries, les souches qui habituellement fermentent le lactose, avec production d'acide et souvent de gaz, sont appelées «coliformes» et comprennent des espèces des genres Citrobacter, Enterobacter, Escherichia et Klebsiella. Cependant, certains médecins microbiologistes incluent les espèces des genres Edwardsiella, Hafnia et 
Serratia, en dépit de leur incapacité habituelle à fermenter le lactose. Certaines souches psychrotrophes, poussent bien à des températures froides, mais montrant une faible inhibition à $37{ }^{\circ} \mathrm{C}$ (Mead, 2007). D'autres souches d'Entérobactéries par exemple, peuvent être impliquées dans l'altération de la viande rouge et la volaille, en particulier dans des conditions de durée de vie prolongée (García-López et al., 1998). Un autre sousensemble du groupe des coliformes comprend les «coliformes fécaux» qui fermentent le lactose à 44,5 $\pm 0,2{ }^{\circ} \mathrm{C}$ et qui sont parfois dénommés «thermo-tolérants». L'espèce la plus fréquemment associée à ce groupe bactérien est Escherichia coli et, dans une moindre mesure le genre Klebsiella (Mead, 2007). Dans les denrées alimentaires d'origine animale, les entérobactéries sont d'origine intestinale ou environnementale et indiquent un défaut d'hygiène lors des processus de fabrication.

\section{Escherichia coli}

Escherichia coli fait partie de la famille des Enterobacteriaceae. Il s'agit de courts bâtonnets mobiles au moyen de flagelles péritriches, Gram négatifs, anaérobies facultatifs, non sporulés, oxydase négative, mesurant de 2 à $4 \mu \mathrm{m}$ de long et d'un diamètre d'environ 0,6 $\mu \mathrm{m}$ (Figure 2). Ils sont capables de fermenter plusieurs sucres, mais leur fermentation du lactose avec production de gaz est caractéristique. La multiplication à 44 ${ }^{\circ} \mathrm{C}$ (optimum $40{ }^{\circ} \mathrm{C}$ et extrême à $45,5^{\circ} \mathrm{C}$ ), la production d'indole et la présence d'une activité ß-glucuronidase, sont également caractéristiques. Les espèces de E. coli sont sérotypées en se basant sur leurs 173 antigènes somatiques $(\mathrm{O}), 56$ antigènes flagellaires $(\mathrm{H})$ et 80 antigènes capsulaires $(\mathrm{K})$ (Feng, 2001 ; Eslava et al., 2003). Etant l'espèce bactérienne anaérobie facultative prédominante dans l'intestin et les fèces, la présence de $E$. coli dans les aliments et l'eau est considérée comme une indication de contamination fécale et, dès lors, l'indication d'une possible présence de microorganismes pathogènes d'origine fécale. La surveillance de E. coli représente le meilleur indicateur d'hygiène des procédés pour suivre la contamination fécale d'un aliment (UE, 2007) mais aucun critère de sécurité n'est pour le moment fixé dans la règlementation Européenne. La contamination a lieu le plus souvent lors de la production et de la transformation d'aliments crus d'origine animale, ou indirectement, via la contamination par de l'eau contaminée (Feng, 2001; Ray, 2001; Eslava et al., 2003). Dans les filières de production carnée, la principale source de contamination des denrées alimentaires par E. coli est le tractus intestinal des animaux. Leur présence indique un défaut de la technique d'abattage, ou une contamination croisée, mais peut également être due à une contamination par les personnes manipulant les denrées alimentaires.

\section{Multiplication de la microflore initiale}

De la mise en vente de la viande à son achat par le consommateur, le nombre de bactéries peut s'accroître par suite du développement bactérien. Cette évolution est fonction du type de conditionnement employé, de la durée de stockage et de la température de conservation. Ainsi, la multiplication de la microflore initiale de la viande peut être inhibée par une conservation de celle-ci à des températures défavorables au développement des microorganismes et par l'application du $\mathrm{pH}$ et d'une activité de l'eau $\left(\mathrm{a}_{\mathrm{w}}\right)$ défavorables. Sont aussi employés, des conservateurs, des microorganismes antagonistes de ceux que l'on cherche à inhiber et le conditionnement sous atmosphère modifiée.

\section{Phases de multiplication des microorganismes}

Sans inhibiteur et avec des conditions environnementales (température, $\mathrm{pH}$, activité de l'eau) favorables, la croissance des microorganismes introduits dans un milieu de culture ou dans un produit alimentaire se déroule en plusieurs phases (Leyral et Vierling, 2007 ; Bailley et al., 2012) :

- Phase de latence : elle est facultative et suit immédiatement l'ensemencement du 
microbe dans le milieu de culture ou l'introduction du microbe dans un produit alimentaire. Il s'agit d'une période d'adaptation au cours de laquelle la cellule synthétise en particulier les enzymes qui lui sont nécessaires pour métaboliser les substrats présents.

- Phase d'accélération: la bactérie se divise de plus en plus activement. La vitesse spécifique de croissance augmente avec le temps.

- Phase logarithmique ou exponentielle de croissance : elle correspond, notamment pour les bactéries à taux de croissance élevé, à un fort potentiel de multiplication, de synthèse d'enzymes et de toxines, de dégradation et d'altération et par voie de conséquence, à un risque considérable. Elle est caractérisée par un rythme de reproduction rapide. Tout au long de cette phase, le temps de génération ou temps de doublement de la population microbienne est constant et minimal. Sa valeur varie d'un microorganisme à un autre et pour un micro-organisme donné, en fonction des conditions environnementales.

- $\quad$ Phase de ralentissement: La vitesse spécifique de croissance diminue progressivement jusqu'à être nulle. Elle est due à la raréfaction des nutriments et correspond à l'accumulation simultanée des produits d'excrétion du métabolisme, certains pouvant être inhibiteurs.

- Phase stationnaire : La concentration cellulaire maximale est atteinte et reste constante. Les cellules conservent une activité métabolique et leur structure biochimique subit des modifications.

- Phase de décroissance: la concentration en cellules viables diminue du fait de la mortalité dont le taux augmente progressivement. Des phénomènes d'autolyse sont dus à l'action des enzymes cellulaires. La structure et la texture des produits naturels et alimentaires conditionnent à la fois la répartition de la contamination et la croissance microbienne. Les microorganismes qui viennent au contact des surfaces musculaires peuvent soit, rester à l'état latent, soit mourir progressivement, soit se multiplier.

\section{Les facteurs de développement}

Le développement de la microflore initiale est influencé par : le nombre initial de microorganisme présent sur la carcasse (principalement les germes d'altération), les espèces ou les souches de germes présentes, la température de conservation, le $\mathrm{pH}$ (lié au taux de glucose dans la viande) particulièrement pour les viandes conservées sous vide ou sous atmosphère modifiée et l'humidité relative qui influence uniquement les viandes non emballées (Corry, 2007). Les plus importants sont le $\mathrm{pH}$, la température, l'activité de l'eau $\left(\mathrm{a}_{\mathrm{w}}\right)$, l'humidité relative et le potentiel d'oxydoréduction $(\mathrm{rH})$ (Lawrie et Ledward, 2006).

\section{Activité de l'eau}

L'activité de l'eau mesure la disponibilité en eau du milieu dans lequel se trouve la microflore. D'une manière générale, plus l'a $\mathrm{w}_{\mathrm{w}} \mathrm{du}$ milieu est élevée, c'est-à-dire proche de 1, plus le développement de la microflore est intense. L'a de la viande fraîche est de l'ordre de 0.993; elle est donc favorable à la multiplication de toutes les espèces microbiennes (James et James, 2000). Si la profondeur de la viande conserve une $a_{w}$ élevée, il n'en est pas de même à la surface. Les microorganismes peuvent se trouver dans l'eau, soit sous forme libre dans les couches superficielles de la carcasse, soit dans l'atmosphère environnante. Une faible humidité relative provoque une forte évaporation qui ne sera plus compensée par le passage de l'eau des tissus profonds. L'activité de l'eau diminue et rend le milieu défavorable à la croissance des bactéries comme Pseudomonas, Acinetobacter, Moraxella. Inversement, une forte humidité relative limite l'évaporation de l'eau et facilite le développement des bactéries psychotrophes. L'activité de l'eau des produits à base de viande peut être réduite par le séchage, l'adjonction de sucre ou de sel, l'ajout de matières grasses ou par congélation (Feiner, 2006). 


\section{Température}

Le facteur le plus important qui régit la croissance microbienne est la température. De façon générale, plus la température est grande, plus le taux de croissance est élevé. Beaucoup de micro-organismes de la viande se développent dans une certaine mesure à toutes les températures, de moins de $0{ }^{\circ} \mathrm{C}$ à $65^{\circ} \mathrm{C}$. Les Psychrophiles (psychrotrophes) ont une température optimale entre $-2{ }^{\circ} \mathrm{C}$ et $7{ }^{\circ} \mathrm{C}$, les mésophiles entre $10{ }^{\circ} \mathrm{C}$ et $40{ }^{\circ} \mathrm{C}$ et les thermophiles de 43 à $66{ }^{\circ} \mathrm{C}$ (Lawire et al., 2006). Les basses températures inhibent le développement des microorganismes. Les paliers suivants sont notés (Rosset et al., 1984) :

- $+10{ }^{\circ} \mathrm{C}$ : arrêt de la toxinogénèse de Clostridium botulinum (A et B);

- $\quad+3{ }^{\circ} \mathrm{C}$ : arrêt de tout risque de nocivité liée à la croissance des germes majeurs ou à l'élaboration de toxines (mais certains psychrotrophes se multiplient encore lentement) ;

- $\quad 0{ }^{\circ} \mathrm{C}$ : température souhaitée pour la conservation de la viande sous vide ;

- $\quad-10{ }^{\circ} \mathrm{C}$ à $-18{ }^{\circ} \mathrm{C}$ : croissance persistante des moisissures et levures ;

- $-18{ }^{\circ} \mathrm{C}$ : arrêt de toute multiplication microbienne.

\section{Le potentiel d'oxydoréduction $(\mathrm{rH})$}

Après la mort, le muscle ayant des réserves en oxygène, présente un potentiel d'oxydoréduction $(\mathrm{rH})$ profond, élevé et positif $(+250 \mathrm{mv})$; ce qui est favorable à la multiplication des germes aérobies (James et James, 2000). Ensuite, les réserves en oxygène n'étant plus renouvelées par le sang, le $\mathrm{rH}$ profond diminue très rapidement, devient négatif et en 8 à $10 \mathrm{~h}$ atteint la valeur de $-150 \mathrm{mv}$ (James et James, 2000). Les conditions réductrices ainsi créées dans la profondeur de la viande sont propices au développement des germes anaérobies de la putréfaction. Dans le cas de viande «normales», le pH acide $(5,7)$ s'oppose à leur multiplication, mais il n'en est pas de même pour les viandes DFD (viandes sombres, collantes et sèches) où le $\mathrm{pH}$ reste élevé (6,3$6,7)$. $p H$

Après abattage, le $\mathrm{pH}$ du muscle passe d'un niveau proche de 7,0 dans le muscle vivant, à environ 5,5-5,7 (chez le bovin) dans le muscle de référence, le faux-filet (Cartier, 2007). Cette valeur ne varie plus lorsque la viande est normalement conservée. Les microorganismes sont extrêmement sensibles aux variations de $\mathrm{pH}$. D'une façon générale, on observe que leur vitesse de développement se trouve réduit par tout abaissement de ce paramètre. Les bactéries sont les premières touchées puis viennent les levures et les moisissures. Toute viande de $\mathrm{pH}$ supérieur à 6,0 est plus sujette aux actions microbiennes notamment à la putréfaction, que la viande normale (James et James, 2000).

\section{Conséquences de la multiplication de la microflore initiale}

La multiplication des germes de la contamination initiale peut donner naissance à des quantités de microorganismes viables à l'origine d'altération conduisant à la putréfaction (germes d'altération) ou aux intoxications alimentaires (germes pathogènes).

\section{Altération des viandes}

Plusieurs types d'altérations sont susceptibles d'atteindre la viande selon la température de conservation. Il s'agit des altérations à basse, à moyenne et à forte température.

Altérations à basse température $\left(<\right.$ à $\left.10^{\circ} \mathrm{C}\right)$ ou putréfaction superficielle

Selon la nature de l'atmosphère, deux types d'altérations sont susceptibles d'apparaître sur les viandes conservées à la chambre froide :

- En atmosphère sèche, la multiplication des bactéries est retardée. Par contre, on assiste à une prolifération lente (une semaine ou plus) de moisissures à la surface de la viande (Aspergyllus, Cladosporium, Thamnidium, Rotrichum, Penicillium, Mucor) participant aux réactions d'hydrolyse et d'oxydation des lipides. Des levures (Candida, Monilia, Torula) ont 
également été isolées mais elles requièrent moins d'attention que les moisissures (Bourgeois et al., 1996 ; Fernandes, 2009 ).

En atmosphère humide, les viandes sont envahies en quelques jours par des bacilles Gram négatif. Il s'agit essentiellement de : Pseudomonas, Acinetobacter, Alcaligenes, Flavobacterium, et Enterobacteriaceae. La viande devient brune grisâtre, elle dégage une odeur putride. Cette putréfaction se traduit par l'apparition en surface d'une couche visqueuse résultant de la juxtaposition de cellules microbiennes (putréfaction superficielle) accompagnée d'odeur nauséabonde. Les bactéries responsables sont des Psychrotrophes, essentiellement Pseudomonas et Achromobacter. Dans le cas où la viande est emballée avec facilité de diffusion de l'oxygène, les deux souches bactériennes sont présentes. En l'absence de l'oxygène dans l'emballage, seule la souche Pseudomonas est éliminée (Ndiaye, 2002). La viande s'altère généralement lorsque le nombre de bactéries Pseudomonas spp. atteint $10^{7} / \mathrm{cm}^{2}-10^{8}$ germes $/ \mathrm{cm}^{2}$ (Mead, 2007).

Altérations à température intermédiaire $(10$ à $25^{\circ} \mathrm{C}$ ) ou puanteur d'os

Le refroidissement lent des carcasses conduit à des altérations en surface et en profondeur. Il se développe en surface de nombreuses espèces avec un pourcentage élevé de germes anaérobies facultatifs et en particulier des entérobactéries; les Pseudomonas y deviennent rapidement l'espèce majoritaire avec apparition d'un poissage et d'une odeur nauséabonde.

En profondeur, le phénomène de puanteur d'os, dont l'étiologie est encore mal connue, s'observe au voisinage immédiat des os des membres postérieurs (James et James, 2000; Mead, 2007) et seuls le liquide synovial et la moelle du fémur sont malodorants. Les formes sporulées de Clostridium sont supposées être responsables du phénomène. De nombreuses espèces de Clostridium et de Bacillus ont été isolées (Bourgeois et al., 1996) dans les tissus lésés toujours à des taux assez faible $\left(10^{2}\right.$ à $10^{3}$ germes $/ \mathrm{g}$ ). Ces germes agiraient conjointement avec des enzymes tissulaires dans des réactions d'hydrolyse et seraient responsables de l'apparition de composés volatils malodorants caractérisant la puanteur d'os (Mead, 2007). La seule prophylaxie efficace est la réfrigération rapide des carcasses associées à des conditions d'abattage satisfaisantes.

\section{Altérations à température élevée $\left(25\right.$ à $\left.40^{\circ} \mathrm{C}\right)$ ou putréfaction profonde}

Les températures de 25 à $40{ }^{\circ} \mathrm{C}$ sont les températures ambiantes fréquentes dans les pays tropicaux ou équatoriaux. Dans les masses musculaires internes de carcasses maintenues à ces températures (absence de réfrigération après l'abattage), s'installe une putréfaction profonde. Elle est due au développement rapide des bactéries anaérobies putréfiantes provenant du tractus intestinal des animaux (Fernandes, 2009). Ces germes se multiplient lorsque le $\mathrm{rH}$ a atteint une valeur suffisamment basse (établissement progressif de l'anaérobiose par consommation tissulaire de l'oxygène résiduel soit 8 à 10 heures post mortem) et que la température reste élevée. Les viandes d'animaux fatigués possédant un potentiel redox $(\mathrm{rH})$ rapidement bas et un pH élevé (viandes sombres ou DFD) se putréfient facilement en profondeur. Cette putréfaction se manifeste en premier lieu par la formation de gaz en l'absence de toute mauvaise odeur. Elle est associée à la présence d'un nombre élevé de Clostridium perfringens sous forme végétative (Fernandes, 2009). Dans un second temps, la viande verdit et devient très malodorante à la suite de la multiplication d'espèces encore plus anaérobies: Clostridium histolyticum, Clostridium sporogenes, Clostridium oedematiens. On estime que les premiers signes de putréfaction apparaissent lorsque le nombre de Clostridium perfringens atteint $10^{7}$ germes/g de viande, pour une contamination initiale de $10^{2}$ germes / g, cela correspond à un facteur de multiplication de $10^{9}$ soit approximativement 30 redoublements $\left(2^{30}=10^{9}\right)$. Une réfrigération précoce et rapide permet d'éviter ces 30 redoublements 
fatidiques et le phénomène de putréfaction profonde (Bourgeois et al., 1996). Ces germes protéolytiques provoquent une décomposition anaérobie des protéines suivie d'une dégradation des acides aminés en indole, en scatole, en mercaptans et en ammoniac. Il se forme également divers amines (histamine, putrescine, cadaverine, etc.) dont la présence rend la consommation de la viande dangereuse. Outre les réactions de protéolyse précitées, les microorganismes de la viande provoquent également les réactions de lipolyse à travers l'hydrolyse des triglycérides de réserves et des phospholipides tissulaires.

\section{Toxi-infection alimentaire}

L'Organisation Mondiale de la Santé (OMS) estime que près de $30 \%$ des habitants des pays industrialisés souffrent chaque année d'une toxi-infection alimentaire (Bailly et al., 2012). Tous les cas sont susceptibles d'être provoqués par les viandes. Sur 1032 foyers déclarés en 2010 en France, 22\% ont été associés à la consommation des produits carnés en général (viande de boucherie, volailles, viandes de charcuterie) (Bailly et al., 2012). Aux USA, le nombre de cas de toxiinfections d'origine alimentaire annuel est estimé à 38,6 millions (Ghafir et Daube, 2007). Parmi ces cas, $71,7 \%$ des mortalités survenues seraient dus à des bactéries. Or la majorité de ces germes provient le plus souvent des denrées alimentaires d'origine animale (Bourgeois et al., 1996). En Afrique subsaharienne, les toxi-infections alimentaires ne sont pas rares, mais leurs estimations sont largement sous-évaluées par les autorités sanitaires et leurs origines sont rarement élucidées du fait de la faiblesse des moyens de diagnostic notamment bactériologiques.

Les microorganismes pathogènes isolés dans les viandes sont: Bacillus cerus (taux relativement faible sur les carcasses); Campylobacter spp. (rencontré plus fréquemment dans la volaille et le porc), Escherichia coli et principalement E. coli $\mathrm{O}$ 157 : H7 (retrouvé dans la viande bovine), Listeria monocytogenes (taux plus élevé souvent dans les viandes hachées),
Clostridium botulinium, Staphylococcus aureus, Clostridium perfringens, Salmonella spp., Yersina enterocolitica (rencontré à un taux plus élevé dans la viande du porc), Avian influenza (retrouvé dans les viandes de volailles), les prions (provenant des cerveaux ou autres organes de bovin), les protozoaires tels que Cryptosporidum parvum, Toxoplasma gondii et Trichinosis spiralis (rencontré dans la viande de porc et animaux sauvage) et les cysticerques retrouvés souvent dans les viandes de bovin et de porc (Ghafir et Daube, 2007 ; Fernandes, 2009 ; Bailly et al., 2012). Aeromonas spp. se développe uniquement sur les viandes qui ont été emballées sous vide ou sous atmosphère modifié (Fernandes, 2009). Bacillus anthracis, Mycobacterium tuberculosis et Brucella abortus sont des germes pouvant provenir de l'animal infecté et dont leur ingestion à travers la consommation de viande infectée et parfois pas cuite à cœur entraîne respectivement chez le consommateur la tuberculose, la brucellose et le charbon bactéridien (Warris, 2000 ; Lawrie et Ledware, 2006). Ces maladies ont tendance à être localisées dans certaines régions du monde. De tous ces germes, les plus incriminés dans les toxi-infections alimentaires en Europe et aux USA sont: Salmonella sp. et Campylobacter (European Food Safety Authority, 2006; Ghafir et Daube, 2007).

\section{Conséquences technologiques}

Les conséquences technologiques concernent l'évolution des caractères organoleptiques et des modifications biochimiques de la viande. L'altération des viandes est un phénomène d'apparition progressive. Les premières manifestations de ce phénomène sont discrètes : odeur dite de relent et modification de l'aspect de la viande. Au-delà du seuil $10^{8}$ bactéries $/ \mathrm{cm}^{2}$, la viande se couvre progressivement d'une couche poisseuse et devient grise ou brune (Leyral et al., 2007). L'oxydation des lipides lui confère une odeur de rancissement. Ces phénomènes sont liés à l'activité oxydative et protéolytique de la flore aérobie associée à des 
fermentations réalisées à partir des substrats glucidiques par des bactéries non protéolytiques, microaérophiles ou aéroanaérobies.

Les altérations de la couleur de la viande fraîche dues aux microbes proviennent de diverses origines et peuvent prendre différentes formes. On assiste à un verdissement dû au dihydrogène sulfuré $\left(\mathrm{H}_{2} \mathrm{~S}\right)$ formé par la protéolyse des acides aminés soufrés ou au dioxygène $\left(\mathrm{O}_{2}\right)$ formé par les lactobacilles en présence d'oxygène.

En raison des nombreuses manipulations nécessaires à leur préparation, les viandes découpées sont très exposées aux contaminations bactériennes. Or, le suc musculaire libéré consécutivement au découpage des viandes constitue un milieu propice au développement bactérien pour que les paramètres physiques tels que le $\mathrm{pH}$ ou la température soient favorables aux bactéries. Les viandes découpées sont davantage sensibles à la putréfaction que les carcasses et les grosses unités de découpe. Par ailleurs, plus les viandes découpées sont exposées à des températures élevées et plus les phénomènes de putréfaction seront d'apparition précoce et d'intensité importante.

\section{Caracteristiques des principaux germes pathogènes de la viande \\ Clostridium botulinium}

Clostridium botulinum est un bacille

Gram positif de 4 à $6 \mu \mathrm{m}$ de longueur, aux extrémités arrondies, mobile (ciliature péritriche), anaérobie strict et sporulé (Figure 3 ). Les souches de $C$. botulinum sont très hétérogènes d'après leurs caractères culturaux, biochimiques et génétiques et elles sont divisées en quatre groupes (I à IV). C'est une bactérie mésophile pouvant se multiplier significativement à $15{ }^{\circ} \mathrm{C}$ (Fernandes, 2009). Les spores ont une résistance de $20 \mathrm{mn}$ à 110 ${ }^{\circ} \mathrm{C}$. Il est un agent d'intoxination et d'intoxication alimentaires humaine ou animale. Les toxines botuliniques se divisent en 7 types (A à $\mathrm{G}$ ) selon leurs propriétés immunologiques, chacune étant neutralisée par un sérum spécifique. La toxine botulique est le poison le plus puissant qui existe. La toxine botulique A est la plus active. La dose létale chez un homme adulte est estimée de $100 \mathrm{ng}$ à $1 \mu \mathrm{g}$ par voie orale (AFSSA, 2006a). Le réservoir de $C$. botulinum, comme des autres Clostridium est l'environnement : sol, poussière, sédiments marin ou d'eau douce, eaux souillées, lisiers et occasionnellement le contenu digestif de l'homme et des animaux sains. L'homme ou l'animal s'infecte en ingérant d'aliment contaminé (notamment conserves et semi-conserve mal stérilisées, jambons crus secs, produits de boucherie et fourrages). La durée d'incubation est de 1 à 10 jours, le plus souvent 1 à 3 jours. La maladie se manifeste par des paralysies flasques (oculaires et cardio-respiratoires), des troubles digestifs (nausées, vomissements, diarrhées ou constipations dysphagie) et urinaires, un tarissement de toutes secrétions (et surtout des sécrétions salivaires). La maladie, plus ou moins grave, peut être mortelle en absence de traitement.

\section{Clostridium perfringens}

Clostridium perfringens appartient au groupe II du genre Clostridium et à la famille des Bacillaceae. Il s'agit d'un bacille Gram positif sporulé, tellurique, anaérobie strict, sulfito-réducteurs, immobiles, possédant une capsule de nature polysaccharidique et facile à voir à l'état frais (Figure 4). Cette espèce est thermophile, sa température optimale de croissance étant comprise entre 40 et $45^{\circ} \mathrm{C}$, mais elle est toutefois capable de se développer à des températures comprises entre $15{ }^{\circ} \mathrm{C}$ et $50{ }^{\circ} \mathrm{C}$. L'a $\mathrm{a}_{\mathrm{w}}$ doit être supérieure à 0,93 et le $\mathrm{pH}$ compris entre 5,5 et 8. Les spores thermosensibles de $C$. perfringens résistent 5 minutes à $100{ }^{\circ} \mathrm{C}$ et produisent l'entérotoxine qui est responsable des intoxications alimentaires (Cavalli et al., 2003; Fosse et al., 2004). Le seuil en dessus duquel il y a intoxication est $10^{5} \mathrm{ufc} / \mathrm{g}$ (AFSSA, 2006c). C'est une bactérie tellurique largement répandue dans l'environnement, qui peut contaminer les fourrages et les ensilages. Ce germe ubiquiste est un hôte normal du tube digestif des animaux et de l'homme. La 
viande peut être contaminée au moment de l'éviscération si du contenu de l'intestin entre en contact avec la carcasse (Cavalli, 2003). L'homme se contamine en ingérant des aliments, notamment des produits carnés, contenant des bactéries. Les denrées incriminées sont les préparations à base de viande et en général cuites, conservées à l'abri de l'air (masses importantes, immersion dans un liquide, emballage étanche), refroidies lentement puis réchauffées lentement, ce qui favorise la multiplication des bactéries et la production de toxines (AFSSA, 2006c). Les symptômes apparaissent entre 6 et $24 \mathrm{~h}$, généralement 10 à $12 \mathrm{~h}$, après l'ingestion du repas contaminé. Ils se traduisent surtout par la diarrhée et de violents maux de ventre, parfois de nausées. Le plus souvent, cette affection guérit spontanément en 2-3 jours. Toutefois, des mortalités ont été observées chez des personnes âgées et des jeunes enfants.

\section{Staphylococcus aureus}

Staphylococcus aureus est un germe de la famille des Micrococcaceae. Il s'agit de cocci à coloration de Gram positive, mesurant 0,5 à $1 \mu \mathrm{m}$ de diamètre souvent disposés en grappe, non sporulés, coagulase positive (Figure 5). Cette espèce fait partie des bactéries aéro-anaérobies facultatives, mais préférant le métabolisme aérobie. C'est un germe mésophile, capable de se multiplier entre $4{ }^{\circ} \mathrm{C}$ et $46^{\circ} \mathrm{C}$, de manière optimale à 37 ${ }^{\circ} \mathrm{C}$, pour un $\mathrm{pH}$ allant de 5 à 9 , avec un optimum de 7,2 à 7,6 et une $\mathrm{a}_{\mathrm{W}}$ de 0,86 en aérobiose et 0,90 en anaérobiose. C'est un germe halophile et xérophile car il se développe même en présence de sel et du sucre et survit dans les aliments déshydratés : sa croissance est possible jusqu'à une concentration de $18 \%$ en sel en aérobiose (Fosse et al., 2004, Bailly et al., 2012 ). Au total, $70-80 \%$ des souches produisent des exotoxines (Cavalli, 2003), dont les entérotoxines staphylococciques A, B, C1, C2, $\mathrm{C} 3, \mathrm{D}, \mathrm{E}$ et $\mathrm{H}$, la toxine du choc toxique staphylococcique et les toxines exfoliatives A et $\mathrm{B}$. La toxinogénèse a lieu pendant la phase exponentielle de croissance, pour une température comprise entre $10{ }^{\circ} \mathrm{C}$ et $45^{\circ} \mathrm{C}$ (avec un optimum de 40 à $45{ }^{\circ} \mathrm{C}$ ), un $\mathrm{pH}$ compris entre 5 et 8 , une teneur en $\mathrm{NaCl}$ inférieure à $10 \%$, une $\mathrm{a}_{\mathrm{w}}$ supérieure à 0,86 (Fosse et al., 2004 ). Staphylococcus aureus est un germe commensal de la flore cutanée des animaux et un agent possible de mammite chez les femelles en lactation (le plus souvent sub-clinique chez la vache, pouvant évoluer vers une forme gangréneuse chez la chèvre et la brebis) (Cavalli, 2003). Chez l'homme, il vit dans la cavité nasale, dans les glandes sébacées et sudoripares, dans les bulbes pileux. Le principal site pour les mains est le bout des doigts (en relation avec l'habitude de se gratter le nez). La contamination des viandes est donc possible au moment du dépeçage, de l'ablation de la mamelle et surtout chaque fois qu'il y a un contact direct entre l'homme et la carcasse. Les troubles (nausées, vomissements, diarrhées) peuvent apparaître chez les consommateurs après ingestion d'un aliment contenant les toxines. La dose minimale à ingérer pour provoquer les premiers symptômes reste mal définie. L'entérotoxine staphylococcique étant très stable à la chaleur, la simple cuisson reste inefficace pour assurer la destruction de la toxine et assainir en toute sécurité un produit de viande contaminé. Il va falloir éviter la contamination ou s'opposer à la multiplication du germe et à la sécrétion de la toxine en ne traversant pas la zone dangereuse $\left(20{ }^{\circ} \mathrm{C}-50\right.$ ${ }^{\circ} \mathrm{C}$ ) ou en n'y résidant que fort peu de temps (ne jamais dépasser une heure).

\section{Salmonella}

Les bactéries du genre Salmonella appartiennent à la famille des Enterobacteriaceae. Genre regroupant de petits bacilles, Gram négatif habituellement mobiles par des cils péritriches (Figure 6), mais des mutants immobiles peuvent exister et S. gallinarum est toujours immobile. Ces bactéries mesurent 0,7 à $1,5 \mu \mathrm{m}$ de diamètre, pour 2 à $5 \mu \mathrm{m}$ de longueur et sont aéroanaérobies facultatives, oxydase négatives et nitrate réductase positives. Elles sont 
mésophiles, capables de se développer à des températures comprises entre $5,2^{\circ} \mathrm{C}$ et $47^{\circ} \mathrm{C}$ et de manière optimale entre 35 et $37^{\circ} \mathrm{C}$, à des $\mathrm{pH}$ compris entre 4,5 et 9 et une $\mathrm{a}_{\mathrm{w}}$ supérieure à 0,93 (Fosse et al., 2004). Au sein de la sous espèce Salmonella enterica enterica, il existe plus de 2400 sérotypes différents parmi lesquels certains sont potentiellement pathogènes pour l'homme. Il s'agit de sérotypes ubiquistes qui peuvent être hébergés dans le tube digestif de l'homme, des animaux domestiques et sauvages, des animaux de compagnie et plus particulièrement des volailles pour $S$. enteritidis. En ce qui concerne la viande bovine, $S$. dublin est également souvent incriminée. Cette dernière peut être hébergée dans le tube digestif des bovins et de l'homme. Les intoxications à salmonelles dues aux viandes sont sérieuses tant par le nombre de malades que par la gravité des symptômes. L'ingestion de $10^{1}$ à $10^{11}$ cellules de Salmonella peut déclencher une infection se manifestant par une fièvre à $39{ }^{\circ} \mathrm{C}-40{ }^{\circ} \mathrm{C}$, des douleurs abdominales, des nausées, des vomissements et un syndrome diarrhéique caractérisé par des selles liquides et fétides (AFSSA, 2002). Tous les sérotypes peuvent être impliqués; ils varient avec les pays et les époques. Salmonella non typhoïdiennes provoquent des abcès dans différents tissus, voire une septicémie. Ces germes résistent au $\mathrm{pH}$ acide de l'estomac, entrent en compétition avec la flore normale de l'intestin grêle et franchissent la barrière épithéliale pour proliférer dans les plaques de Peyer et envahir les ganglions mésentériques (Hanes, 2003).

\section{Escherichia coli}

Les germes $E$. coli sont normalement présents parmi la microflore digestive de l'homme et de nombreux animaux à sang chaud, comme par exemple les bovins. La plupart des E. coli sont sans danger pour l'homme et l'animal. Cependant certaines souches sont pathogènes pour l'homme, à l'exemple de Escherichia coli entérohémorragiques ou EHEC (EnteroHemorrhagic E. coli), dont la plus connue est
E. coli O157:H7 et ayant un lien épidémiologique assez étroit avec le bœuf (Fernandes, 2009 ; Bailly et al., 2012).

La principale maladie qu'elles provoquent chez l'homme est la colite hémorragique. Outre la colite hémorragique, les EHEC peuvent causer de la diarrhée, le syndrome hémolytique et urémique (SHU) principalement chez le jeune enfant ou le micro-angiopathie thrombotique (MAT) chez l'adulte (Feng, 2001 ; Ray, 2001). Les EHEC libèrent des toxines, les shigatoxines (encore appelées vérotoxines), qui induisent des lésions de l'endothélium vasculaire, principalement intestinal, rénal et cérébral. Les shigatoxines, Stx1 et Stx2, sont codées par les gènes stx. Toute souche de E. coli possédant un gène stx est appelée $E$. coli producteur de shigatoxine ou STEC (shigatoxin-producing $E$. coli) ou encore VTEC (verotoxin-producing $E$. coli). La prévalence du portage de STEC par les bovins varie en fonction des élevages. Les sources du danger sont les animaux porteurs, les sols contaminés (prairies, champs), les eaux superficielles contaminées par des déjections animales ou d'engrais de fermes, les aliments (herbes, fourrages) et l'eau d'abreuvement des animaux (ANSES, 2011).

Les infections sont le plus souvent causées par la consommation de viande de bœuf contaminée et insuffisamment cuite, mais peuvent également être dues à la consommation d'eau, de lait cru, de fruits, de légumes, à des baignades et à des contacts entre personnes (Feng, 2001). Le temps de réduction décimal est compris entre 0,5 à 3 $\mathrm{mn}$ à $60{ }^{\circ} \mathrm{C}$, mais une augmentation de la thermorésistance est possible si la viande est fortement riche en matière grasse (ANSES, 2011). La relation dose-effet est faible; six bactéries de E. coli O157:H7 par gramme de steak haché de bœuf ingéré ont entraîné une épidémie en France en 2005. Quant à la relation dose-réponse, elle est estimée à 300 à 600 bactéries pour provoquer chez les enfants de 5 à 10 ans le syndrome hémolytique et urémique (ANSES, 2011). 


\section{Yersinia enterocolitica}

Le genre Yersinia comprend 11 espèces appartenant aux Enterobacteriaceae. Il s'agit de bacilles Gram négatifs, non sporulés, anaérobies facultatifs qui fermentent le glucose. Plus petites que la plupart des autres entérobactéries, elles apparaissent souvent comme des coccobacilles (Figure 7) lorsqu'elles se multiplient à $37{ }^{\circ} \mathrm{C}$. Il comprend 4 espèces pathogènes bien caractérisées : Yersinia pestis responsable des pestes bubonique et pulmonaire, $Y$. pseudotuberculosis pathogène des rongeurs et occasionnellement de l'homme, Y. ruckeri provoquant des maladies chez les poissons d'eau douce et $Y$. enterocolitica, un pathogène intestinal. $Y$. pseudotuberculosis et $Y$. enterocolitica sont les 2 agents pathogènes d'origine alimentaire. Elles atteignent le tractus gastro-intestinal de l'homme et provoquent des entérites, entérocolites, lymphadénites, et rarement des infections extra-intestinales telles que des arthrites. $Y$. enterocolitica est également présente dans l'intestin d'animaux sains tels que des porcs, des bovins, des chiens et des chats (Krauss et al., 2003; Robin-Browne et Hartland, 2003). L'espèce $Y$. enterocolitica est divisée en plusieurs sous-groupes suivant leur activité biochimique et les antigènes $\mathrm{O}$ lipopolysaccharides (sérotype) qu'ils portent. Les sérotypes impliqués dans les pathologies humaines sont: sérotype 0:3 (en Europe), sérotypes O:8, O:5 et O:27 (USA, Canada ou Japon). Y. enterocolitica est psychrotrophe, c'est-à-dire capable de se multiplier à des températures inférieures à $4{ }^{\circ} \mathrm{C}$. $\quad \mathrm{Sa}$ température optimale de multiplication est cependant de $28-30{ }^{\circ} \mathrm{C}$ (Krauss et al., 2003; RobinBrowne et Hartland, 2003). $Y$. enterocolitica est présente chez plusieurs espèces d'animaux, dans les aliments et dans les eaux. Mais les porcs sont le principal réservoir des biosérotypes pathogènes pour l'homme. La région des amygdales de porcs est une niche écologique ayant une haute incidence de $Y$. enterocolitica. Pour $Y$. enterocolitica, les symptômes cliniques se manifestent classiquement chez l'adulte par une entérocolite avec la triade : Fièvre, crampes abdominales, diarrhée liquide aigüe, pouvant s'accompagner de céphalées et d'anorexie ou de vomissements. Chez l'enfant, on constate plutôt une diarrhée aqueuse et muqueuse. La dose minimale infectante est de l'ordre de $10^{6}$ microorganismes (AFSSA, 2006b). Les températures de pasteurisation détruisent les bactéries de Yersina entéropathogènes. Ainsi, les principaux couples temps/températures dans les lignes directrices de production pour la cuisson des viandes sont : pour la cuisson, viandes hachés de bœuf, veau, agneau, porc (15 secondes à $71{ }^{\circ} \mathrm{C}$ à cœur du produit), viandes hachées de volailles (15 secondes à $74^{\circ} \mathrm{C}$ à cœur du produit), viandes coupées de bœuf, veau, agneau, jambon (15 secondes à $63{ }^{\circ} \mathrm{C}$ à cœur du produit) et pour le stockage des aliments (à cœur du produit : $<5{ }^{\circ} \mathrm{C}$ pendant 7 jours ; $<7.2{ }^{\circ} \mathrm{C}$ pendant 4 jours) (AFSSA, 2006b).

\section{Campylobacter}

Le genre Campylobacter est constitué de fins bacilles Gram négatifs incurvés en spirale, non sporulés, parfois en forme de $S$ (doté d'un flagelle polaire, non entouré d'une gaine, situé à l'une des extrémités ou aux deux extrémités, lui conférant cette forme effilée), d'une taille de 0,2 à $0,9 \mu \mathrm{m}$ de diamètre et de 0,5 à $5 \mu \mathrm{m}$ de long (Figure 8) (ASPC, 2012). Campylobacter a un métabolisme de type respiratoire et est micro-aérophile. Certaines souches peuvent occasionnellement se multiplier dans des conditions d'aérobiose ou d'anaérobiose. Ils sont incapables d'oxyder ou de fermenter les sucres et sont positifs au test de l'oxydase (Ghahir et Daube, 2007).Toutes les espèces de Campylobacter se multiplient à $37{ }^{\circ} \mathrm{C}$, mais les Campylobacter thermophiles (C. jejuni, $C$. coli et $C$. lari) ont une meilleure croissance à $42{ }^{\circ} \mathrm{C}$ et ne se multiplient pas à une température inférieure à $25{ }^{\circ} \mathrm{C}$. Ces bacilles sont plus sensibles aux conditions défavorables, telles que la dessiccation, la chaleur, l'acidité, les désinfectants ou l'irradiation, que la plupart d'autres bactéries 
pathogènes intestinales (Hu et Kopecko, 2003). C. jejuni et C. coli causent plus de $95 \%$ des campylobactérioses. Le réservoir est le tractus intestinal des animaux domestiques et sauvages, particulièrement les oiseaux (Butzler, 2004). La transmission a lieu généralement par la consommation d'aliments (viande de volaille insuffisamment cuite), d'eau, des contacts directs ou la manipulation d'animaux infectés (animaux de boucherie et de compagnie) (Hu et Kopecko, 2003). Les mécanismes de virulence de Campylobacter ne sont pas encore bien connus. Ils auraient comme composantes des toxines, l'adhérence, la mobilité, la capacité de capter le fer et l'invasion bactérienne. La campylobactériose donne lieu à de la fièvre, de la diarrhée et de fortes douleurs abdominales. La guérison a généralement lieu sans traitement, après 2 à 6 jours. Des infections extra intestinales sont décrites dans 1,5 cas/1000 infections intestinales. Les conséquences peuvent en être le syndrome de Guillain-Barré (une polyneuropathie inflammatoire aigüe résultant en une paralysie neuromusculaire) et le syndrome de Reiter (une arthropathologie impliquant de multiples articulations) ( $\mathrm{Hu}$ et Kopecko, 2003; Butzler, 2004). La dose infectieuse varie de 500 à 900 bactéries (ASPC, 2012). Campylobacter est fréquemment présent dans le tractus intestinal des volailles, porcs et bovins, mais en raison des techniques d'abattage de cette espèce, la viande de volaille est la principale source de contamination de l'homme (Jorgensen et al., 2002). Elle est inactivée par la chaleur à $70^{\circ} \mathrm{C}$ pendant 1 minute.

\section{Normes microbiologiques de la viande}

L'objet des normes microbiologiques de la viande ou tout autre aliment est de protéger la santé du consommateur et de normaliser les transactions commerciales. Les critères microbiologiques applicables à la viande crue existent mais varient d'un continent ou d'un pays à un autre. Toutefois, il existe des organisations internationales telles que le Codex Alimentarius (CCA), l'Organisation des Nations-Unies pour l'Alimentation et l'Agriculture (FAO), l'Organisation Mondiale de la Santé (OMS), la Commission Internationale des Spécifications Microbiologiques pour les aliments (ICMSF), l'Organisation Internationale de Normalisation (ISO), l'Organisation Mondiale de Santé Animale (OIE), la Commission de l'Union Européenne (UE) et l'Agence Standard de l'Alimentation (FSA) qui élaborent des règlements et s'engagent dans l'application de ces derniers dans le but de garantir la sécurité publique et le commerce mondial des aliments. A titre d'exemple, en Europe, le règlement $(\mathrm{CE}) \mathrm{N}^{\circ}$ 2073/2005 de la Commission Européenne du 15 novembre 2005 (UE, 2005) modifié par le règlement (CE) No 1441/2007 de la Commission Européenne du 5 décembre 2007 (UE, 2007) est appliquée pour les critères de sécurité relatifs à l'acceptabilité des denrées alimentaires et des critères d'hygiène du procédé indiquant l'acceptabilité d'un procédé de production. Aux Royaumes Unis (UK), ce même règlement a été adopté en 2006 (Food Law, 2012). Bien que ces règlements de l'UE d'hygiène alimentaire soient directement applicables, chaque État membre a la possibilité d'adopter certaines dispositions dans sa législation nationale. Aux Etats-Unis, toutes les réglementations en vigueur par rapport aux aliments sont répertoriées dans le Code of Federal Regulations (Lawley, 2007). En Inde, les règlements de contaminants microbiens sont sous l'égide de la Prévention des Règles de l'Altération des aliments (PFA, 1954) amandé en 2004 (Biswas et al., 2011). En Afrique francophone, sont utilisées comme références, les normes du Codex Alimentarius et celles de l'Union Européenne. 


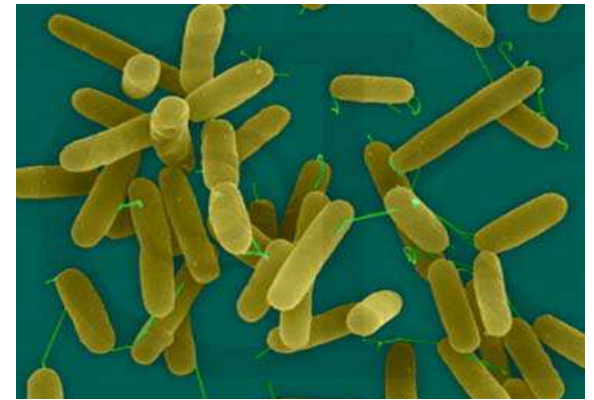

Figure 1: Pseudomona aeruginosa (Winsor et al., 2011).

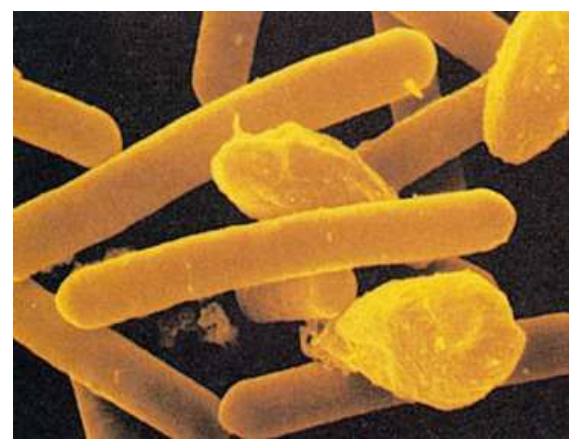

Figure 3: Clostridium botulinium (Burne, 2012).

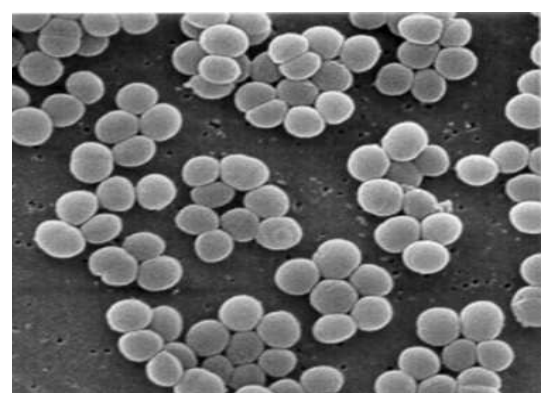

Figure 5: Staphylococcus aureus (Matthew et Carr, 2012).

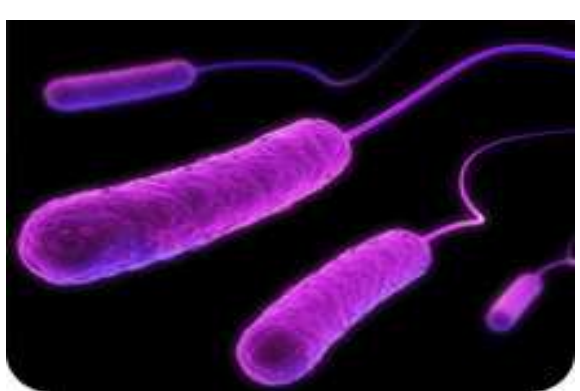

Figure 2: E. coli (Canadien en Santé, 2012).

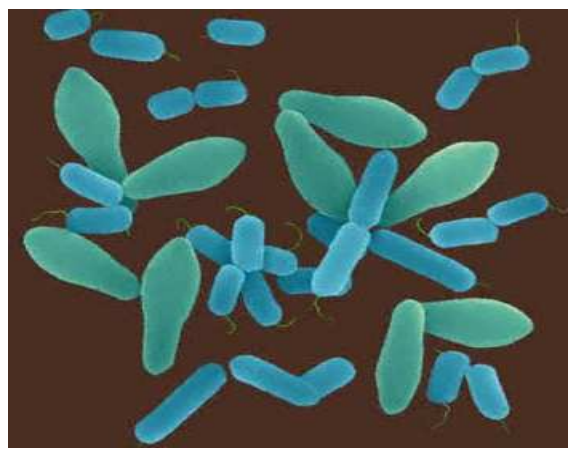

Figure 4: Clostridium perfringens (Kunkel, 2008).

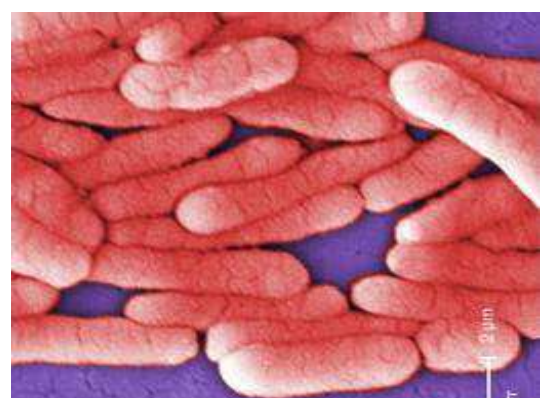

Figure 6: Salmonella (Mousset, 2011). 


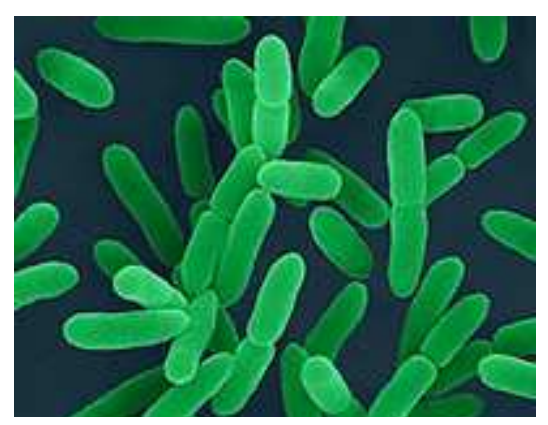

Figure 7: Yersina Enteroitica (Kunkel, 2004)

\section{Conclusion}

Le processus de transformation des animaux vivants en viande, entraîne inévitablement une contamination microbienne de surface des carcasses. La plupart des micoorganismes transférés aux carcasses pendant le processus d'abattage sont des agents pathogènes. Au nombre de ceux-ci, on peut citer: Bacillus cerus, Campylobacter, Escherichia coli O 157: H7, Listeria monocytogenes, Clostridium botulinium, Staphylococcus aureus, Clostridium perfringens, Salmonella spp. et Yersina enterocolitica. Ces agents pathogènes de la viande font l'actualité sur le plan de la sécurité alimentaire dans presque tous les pays $\mathrm{du}$ monde. Les risques associés à leur consommation sont cependant différents en raison des différences culturelles dans la consommation alimentaire et dans les habitudes des conditions de traitement.

\section{REFERENCES}

AFSSA (Agence Française de Sécurité Sanitaire des Aliments). 2002. Fiche de description de dangers transmissibles par les aliments: Salmonella spp. AFSSA, 6p. http://www.infectiologie.com

AFSSA (Agence Française de Sécurité Sanitaire des Aliments). 2006a. Fiche de description de dangers transmissibles par

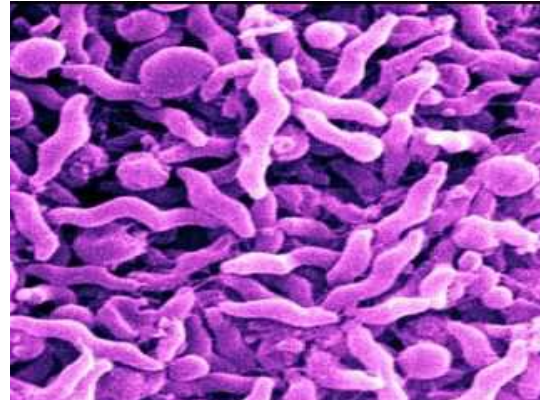

Figure 8: Campylobacter (Chuck, 2013).

les aliments: Clostridium botulinum. AFSSA, 4p. http://www.afssa.

AFSSA (Agence Française de Sécurité Sanitaire des Aliments). 2006b. Fiche de description de dangers transmissibles par les aliments: Yersinia enterocolitica, Yersinia pseudotuberculosis. Agent de la yersiniose, pseudotuberculose. AFSSA, 4p. http://www.afssa.

AFSSA (Agence Française de Sécurité Sanitaire des Aliments). 2006c. Fiche de description des dangers transmissibles par les aliments : Clostridium perfringens. Agent de toxi-infection alimentaire. AFSSA, 4p. http://www.infectiologie.com

ANSES (Agence Nationale de Sécurité Sanitaire de l'Alimentation, de l'Environnement et du Travail). 2011. Fiche de description de danger biologique transmissible par les aliments. E. coli enterohémorragiques (EHEC). ANSES, 4p. http://www.anses.fr/ Documents/MIC-Fi-EscherichiaColi.pdf

ASPC (Agence De Sante Publique Canada). 2012. Campylobacter coli; fiche technique santé-sécurité : agents pathogènes. ASPC. http://www.phacaspc.gc.ca/lab-bio/res/psdsftss/campylobacter-coli-fra.

Bailly JD, Brugere H, Chadron H. 2012. Microorganismes et Parasites des 
Viandes: les Connaître pour les Maîtriser de l'Eleveur au Consommateur. CIV, 150p. www.civViande.org.

Benaissa A. 2011. Etude de la qualité microbiologique des viandes camelines et ovines conservées selon différents modes. Mémoire en vue de l'obtention du diplôme de Magister en Biologie, Option Microbiologie Appliquée, Université Kasdi Merbah Ouargla, Faculté des Sciences de la Nature et de la Vie et des Sciences de la Terre et de l'Univers, Département des Sciences de la Nature et de la Vie, 61p.

Biswas AK, Kondaiah N, Anjaneyulu ASR, Mandal PK. 2011. Causes, concerns, consequences and control of microbial contaminants in Meat. Int. J. of Meat Sci., 1(1): 27.35 .

Bonnefoy C, Guillet F, Leyral G, VernesBourdais E. 2002. Population contaminant altérant la qualité sanitaire et marchande. In Microbiologie et Qualité dans les Industries Agroalimentaires. Collection Biosciences et Techniques, Série Sciences des Aliments; 248p.

Bourgeois CM, Mescle JF, Zucca J. 1996. La microflore de la viande (336-345). In Microbiologie Alimentaire: Aspect Microbiologique de la Sécurité et de la Qualité des Aliments. Lavoisier Tec \& Doc: Paris; 672 p.

Burne A. 2012. Clostridium botulinum, Food Safety, FSA, Outbreak. http://www. befoodsafe.org.uk

Butzler JP. 2004. Campylobacter, from obscurity to celebrity. Clin. Microbiol. Infect., 10: 868-876.

Cartier P, Moevi I. 2007. Le point sur la qualité des carcasses et des viandes de gros bovins. Département Techniques d'Elevage et qualité. Service Qualité des viandes. Compte rendu final $\mathrm{n}^{\circ} 1705$ 32 022, 70p.

Cartier P. 2004. Points de Repères en Matière de Qualité Microbiologique Viandes Bovines. Collection Interbev ; 179p.

Cavalli S. 2003. Application de la méthode HACCP en établissement d'abattage : modèles théoriques et essai de mise en place. Thèse de Médecine Vétérinaire, ENVL, Lyon, 132p.

Chuck J. 2013. Campylobacter Fact Sheet. Gass Weber Mullins LLC. http://www.defendingfoodsafety.com/foo d-safety-law/common-food-bornepathogens/campylobacter-1/

Coibion L. 2008. Acquisition des qualités organoleptiques de la viande bovine: adaptation à la demande du consommateur. Mémoire pour l'obtention du grade de Docteur Vétérinaire, Ecole Nationale Vétérinaire de Toulouse, $97 \mathrm{p}$.

Corry TEL. 2007. Spoilage organisms of red meat and poultry (101-122). In Microbiological Analysis of Red Meat, Poultry and Eggs, Mead GC (Ed). Woodhead publishing limited and CRC press LLC: Cambridge, England; 348p.

Dennaï N, Kharrattib B, El Yachiouim A. 2001. Appréciation de la qualité microbiologique des carcasses de bovins fraîchement abattus. Ann. Méd. Vet., 145: 270-274.

EFSA. 2013. Food-borne zoonotic diseases. EFSA, http://www.efsa.europa.eu/fr/ topics/topic/foodbornezoonoticdiseases.h tm

Eslava C, Villaseca J, Hernandez U, Cravioto A. 2003. Escherichia coli (123-135). In International Handbook of Foodborne Pathogens, Miliotis MD, Bier JW (eds). Marcel Dekker: New York; 688p.

European Food Safety Authority 2006.The community summary report on trends 
and sources of zoonoses, zoonotic agents, antimicrobial resistance and foodborne outbreaks in the European Union in 2005. The EFSA Journal, 94: 1236.

Euzeby JP. 2007. Dictionnaire de bactériologie vétérinaire. Adresse URL, http://www.bacterio.cict.fr/bacdico/, consulté le 15/08/2012.

Feiner G, 2006. Definitions of terms used in meat science and technology (46-71). In Meat Products Handbook Practical Science and Technology. Wood head publish inglimited and CRC Press LLC: Cambridge, England; 629p.

Feng P. 2001. Escherichia coli (143-162). In Guide to Foodborne Pathogens, Labbé RG, García S (Eds). John Wiley and Son: New York; 400p.

Fernandes R. 2009. Chilled and frozen raw meat, poultry and their products (1-52). In Microbiology Handbook Meat Products. Leatherhead Publishing, Randalls Read, Leatherhead, surrey KT22 7RY, UK and Royal Society of Chemistry, Thomas Graham House, Science Park Milton Road: Cambridge; $297 \mathrm{p}$.

Food Law. 2012. Practice Guidance. England. Food Law, 24p. Consulté le 04 Avril 2013 à l'adresse: http://www.food.gov .uk/multimedia/pdfs/practiceguidanceeng .pdf

Fosse J, Cappelier J-M, Laroche, Fradin N, Giraud K, Magras C. 2006.Viandes bovines: une analyse des dangers biologiques pour le consommateur appliquée à l'abattoir. Ren. Rech. Rum., 13: 411-414.

Fosse J, Margas C. 2004. Dangers Biologiques et Consommation des Viandes. Ed Lavoisier: Paris; 220p.

García-López ML, Prieto M, Otero A. 1998. The physiological attributes of Gram- negative bacteria associated with spoilage of meat and meat products (134). In The Microbiology of Meat and Poultry, Davies A, Board R (eds). Blackie Academic and Professional: London UK; 247p.

Ghafir Y, Daube G. 2007. Le point sur les méthodes de surveillance de la contamination microbienne des denrées alimentaires d'origine animale. Ann. Méd. Vét., 151: 79-100.

Hanes D. 2003. Nontyphoid Salmonella (137149). In International Handbook of Food-borne Pathogens, Miliotis MD, Bier JW (eds). Marcel Dekker: New York; 688p.

$\mathrm{Hu}$ L, Kopecko DJ. 2003. Campylobacter Species (181-198). In International Handbook of Foodborne Pathogens. Marcel Dekker: New York; 688p.

James SJ, James C. 2000. Microbiology of refrigerated meat (3-19). In Meat Refrigeration. Wood head publishing limited and CRC press LLC: Cambridge England; 347p.

Jorgensen F, Bailey R, Williams S, Henderson $\mathrm{P}$, Wareing DR, Bolton FJ, Frost JA, Ward L, Humphrey TJ. 2002. Prevalence and numbers of Salmonella and Campylobacter spp. on raw, whole chickens in relation to sampling methods. Int. J. Food Microbiol., 76: 151-164.

Kpodekon TM, Goussanou JSE, Attakpa YE, Boko CK, Ahounou GS, Salifou CFA, Tougan PU, Youssao AKI. 2013. Evaluation of macroscopic and microbiological hazards of indigenous pork consumption in south of Benin. Int. J. Curr. Microbiol. App. Sci., 2(5): 98109.

Krauss H, Weber A, Appel M, Enders B, Isenberg HD, Schiefer HG, Slenczka W, Vongraevenitz A, Zahner H. 2003. 
Zoonoses: Infectious Diseases Transmissible from Animals to Humans. ASM Press: Washington; 456 p.

Kunkel D. 2004. Microscopy Inc. In What is Yersinia enterocolitica? EHA Consulting Group, Inc. Consulté le 09 Mai 2013 à l'adresse : http://www.ehagroup.com/ resources/pathogens/yersiniaenterocolitica/

Kunkel D. 2008. Clostridium perfringens. Musée Armand-Frappier ; Consulté le 21 Avril 2013 à l'adresse : http://www.museeafrappier.qc.ca/fr/inde $\mathrm{x}$.

Lawley R. 2007. Food safety legislation. Consulté le 04 Avril 2013 à l'adresse www.foodsafetywatch.com/

Lawrie RA, Ledward DA. 2006. The spoilage of meat by infecting organism (157188). In Lawrie's Meat Science ( $7^{\text {th }}$ edition). Woodhead Publishing Limited, Abington Hall, Cambridge CB1 6AH: England, Abington; 442p.

Layeral G, Vierling E. 2007. Physiologie du monde bactérien (37-66). In Microbiologie et Toxicologie des Aliments : Hygiène et Sécurité Alimentaire. Sciences des Aliments. Ed. Rueil-Malmaison Doin ; Bordeau CRDP d'Aquitaine; 290p.

Matthew JA, Carr J. 2012. Staphylococcus aureus. CDC Public Health Image Library. Consulté le 21 Avril 2013 à l'adresse: http://commons.wikimedia. org/wiki/File:Staphylococcus_aureus_01 .jpg

Mead C. 2007. Microbiological Analysis of Red Meat, Poultry and Eggs. Published Woodhead Limited and CRC press, ambridge CB21 6AH, LLC: England; $335 \mathrm{p}$.

Mousset A. 2011. Salmonelles : émergence de multi-résistantes. Procès Alimentaire, Magasine de l'industrie agroalimentaire.
Consulté le 21 Avril 2013 à l'adresse : http://www.processalimentaire.com/Qual ite/Salmonelles-emergence-de-multiresistantes-18701

Ndiaye ML. 2002. Contribution à l'étude de la contamination microbiologique de la viande des volailles. Mémoire de Physique appliquée à la biologie, Dakar, $23 \mathrm{p}$.

Ray B. 2001. Indicators of bacterial pathogens (409-417). In Fundamental Food Microbiology, Ray B (ed). CRC Press: Boca Raton; 355p.

Robin-Browne RM, Hartland EL. 2003. Yersinia species (323-355). In International Handbook of Foodborne Pathogens.: Marcel Dekker: New York; $688 \mathrm{p}$.

Rosset R, Lameloise P, Dumont B. 1984. Microbiologie de la viande (131-189). In Les Viandes. SOCOPA : Paris ; 645p.

Rosset R. 1978. L'hygiène dans l'alimentation collective ou les 3 règles et les 10 commandements de la lutte anti TIAC. La Revue Générale $d u$ Froid. Cuisine collective, 69: 29-30.

Salifou CFA, Youssao AKI, Ahounou GS, Tougan PU, Farougou S, Mensah GA, Clinquart A. 2013. Critères d'appréciation et facteurs de variation des caractéristiques de la carcasse et de qualité de la viande bovine. Rapport de point de thèse, Université d'AbomeyCalavi, Abomey-Calavi, 125p.

UE. 2005. Union Européenne, règlement (CE) $n^{\circ} 2073 / 2005$ de la Commission du 15 novembre 2005 concernant les critères microbiologiques applicables aux denrées alimentaires. Journal Officiel de l'Union Européenne, L $338 / 24$.

UE. 2007. Règlement (CE) $\mathrm{N}^{\circ} 1234 / 2007$ du Conseil du 22 octobre 2007 portant organisation commune des marchés dans 
le secteur agricole et dispositions spécifiques en ce qui concerne certains produits de ce secteur (règlement $\mathrm{OCM}$ unique) JO L 299 du 16.11.2007, L 299/1- L 299/149.

Warris DP. 2000. Meat Hygiene, Spoilage and Preservation (182-205). In Meat Science An Introductory Text. CABI Publishing, School of Veterinary Science, University of Bristol: Bristol, UK; 29p.
Winsor GL, Lam DK, Fleming L, Lo R, Whiteside MD, Yu NY, Hancock RE, Brinkman FS. 2011. Pseudomonas Genome Database: improved comparative analysis and population genomics capability for Pseudomonas genomes. Nucleic Acids Res., 39: 596600 . 